\title{
Magnetooptical Effects at Submillimetre Wavelengths Near AFMR in $\mathrm{YFeO}_{3}$
}

\author{
A.A. Mukhin, V.D. Travkin, S.P. Lebedev, A.S. Prokhorov, A.M. Balbashov* and I. Yu. Parsegov* \\ General Physics Institute of the Russian Academy of Sciences, 38 Vavilov St., 117942 Moscow, Russia \\ * Moscow Power Engineering Institute, 14 Krasnokazarmennaya St., 105835 Moscow, Russia
}

\begin{abstract}
Spectra of the transmission $T(v)$, ellipticity $\rho(v)$ and Faraday rotation angle $\alpha F(v)$ have been measured in $\mathrm{YFeO}_{3}$ single crystals near AFMR mode $\left(v_{1} \approx 10 \mathrm{~cm}^{-1}\right)$ using the quasi-optical submillimetre backward-wave-oscillator spectrometer. A resonance behaviour of the $T(v), \rho(v)$ and $\alpha_{F}(v)$ spectra was observed near AFMR. The theoretical calculations and computer simulations made it possible to describe the observed $T(v), \rho(v)$ and $\alpha F(v)$ spectra and to determine both diagonal and non-diagonal components of the permittivity and permeability of the $\mathrm{YFeO}_{3} . \mathrm{An}$ unusual effect of the weak magnetic field $(\mathrm{H} \approx 250 \mathrm{Oe})$ along magnetic moment (m/lc-axis) on the line-shape and intensity of the resonance line was revealed and explained by an interference of two normal modes inside the resonance line.
\end{abstract}

\section{INTRODUCTION}

Rare-earth $(\mathrm{R})$ orthoferrites $\mathrm{RFeO}_{3}$ have proved to be an interesting class of weak ferromagnets for investigations of various magnetic interactions, phase transitions, magnetooptical properties and magnetic excitations. A diverse and unusual behaviour of the antiferromagnetic resonance (AFMR) modes was observed in orthoferrites at submillimetre wavelengths which is determined by dynamic properties both Fe and R-subsystems and their exchange interaction [1].

Also unusual magnetooptical effects are expected near resonance frequencies of the magnetic excitation (for example, Faraday rotation [2]). In this work we have studied the propagation of the plane-polarized electromagnetic waves along the magnetization direction and related magnetooptical effects in the yttrium orthoferrites $\left(T_{N}=648 \mathrm{~K}\right.$ ) as a model crystal for investigation of corresponding effects in the Fe-subsystem only.

\section{EXPERIMENTAL RESULTS}

Single crystals of the $\mathrm{YFeO}_{3}$ were grown by the floating zone method with radiative heating. The transmission spectra $T(v)$ of the $0.43 \mathrm{~mm}$ thick c-cut plane-parallel plate of the $\mathrm{YFeO}_{3}$ were measured using the quasi-optical submillimetre backward-wave-oscillator spectrometer [1] in the frequency range 7.7-13 $\mathrm{cm}^{-1}$ near the resonance frequency of the AFMR mode $\left(v_{1} \approx 10 \mathrm{~cm}^{-1}\right)$ excited by the $\mathbf{h} \| \mathbf{a}-$ and $\mathbf{h} \| \mathbf{b}$-axes. Besides the $\mathrm{T}(v)$ spectra we were able to obtain also the spectra of ellipticity $\rho(v)$ and the Faraday rotation angle $\alpha_{F}(v)$ (the rotation angle of the principle axis of a polarization ellipse) by the measurements at different orientation $\alpha_{A}$ of an analyser with respect to a polarization plane of the incident radiation.

Examples of the $T(v)$ spectra in the case of $h \| b$, enla polarization of the incident radiation and various $\alpha_{A}$ are shown in the Fig.1. The measurements were performed in the weak magnetic field $(\mathrm{H} \approx 250 \mathrm{Oe})$ along magnetic moment $(\mathrm{H}|| \mathrm{m} \| \mathrm{c}$-axis) in order to remove the domain structure. A strong increase of the transmission near AFMR line at $10 \mathrm{~cm}^{-1}$ in the case of $\alpha_{A}=90^{\circ}$ as well as change of the line-shape and its intensity for $\alpha_{A}=0^{\circ}$ and $45^{\circ}$ indicate noticeable rotation of the radiation polarization plane. In order to determine the spectra of ellipticity $\rho(v)$ and Faraday rotation angle $\alpha_{F}(v)$ we used transmission $T_{0}, T_{45}$ and $T_{90}$ spectra corresponding to $\alpha_{A}=0^{\circ}, 45^{\circ}$ and $90^{\circ}$, respectively, and following relations:

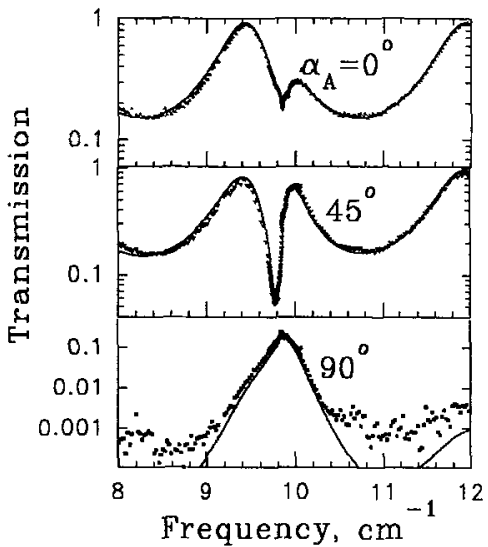

Fig.1. Transmission spectra at e\|la, $h \| b$ and various orientation $\alpha_{A}$ of the analyser (here and in other figures points denote experiment, and lines theory). 


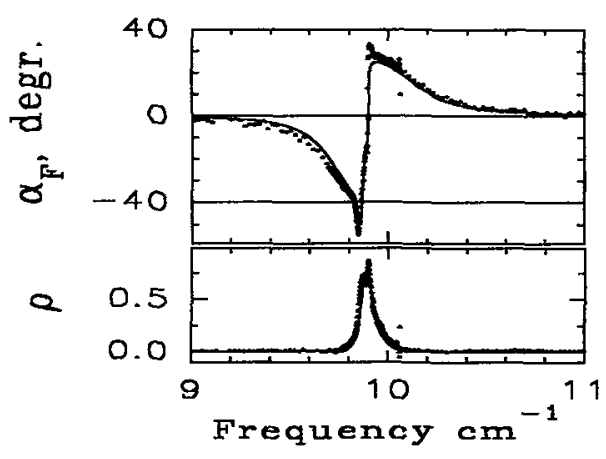

Fig.2. Spectra of the Faraday rotation angle $\alpha_{F}(v)$ and the ellipticity $\rho(v)$ for $e\|a, h\| b$.

$$
\operatorname{tg} 2 \alpha_{\mathrm{F}}=\left(\mathrm{T}_{45}-\mathrm{T}_{0}-\mathrm{T}_{90}\right) /\left(\mathrm{T}_{0}-\mathrm{T}_{90}\right),
$$

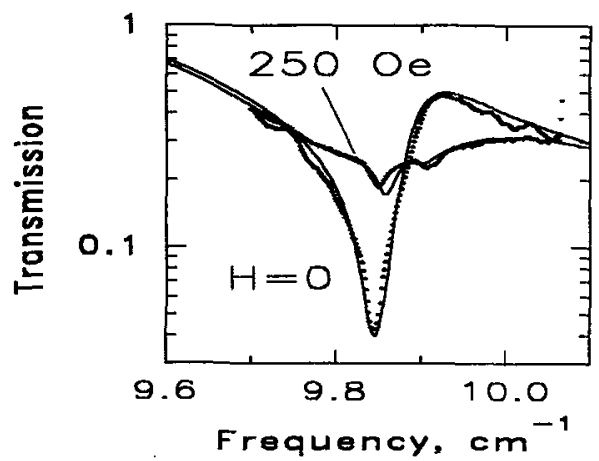

Fig.3. Transmission spectra near AFMR line for magnetized $(\mathrm{H}=250 \mathrm{Oe})$ and demagnetized $(\mathrm{H}=0)$ states of the $\mathrm{YFeO}_{3}$ for $\mathrm{e}\|\mathrm{a}, \mathrm{h}\| \mathrm{b}, \alpha_{\mathrm{A}}=0$.

$\rho \equiv\left(\mathrm{E}_{\min } / \mathrm{E}_{\max }\right)^{2}=(1-\delta) /(1+\delta)$,

where $\delta=\left[1-4 T_{0} T_{90} \sin ^{2} \Delta \varphi /\left(T_{0}-T_{90}\right)^{2}\right]^{1 / 2}, \cos \Delta \varphi=\left(T_{45}-T_{0}-T_{90}\right) / 2\left(T_{0} T_{90}\right)^{1 / 2}, \Delta \varphi=\varphi x-\varphi$ is a phase shift between $x$ - and $y$-component of the transmitted electric field $\mathbf{E}$, which coincide with a- and $b$-axis respectively, $E_{\text {min }}$, $E_{\max }$ are transmitted electric field along small and large axis of a polarization ellipse respectively. The corresponding $\alpha_{F}(v)$ and $\rho(v)$ spectra obtained in such a manner (Fig.2) demonstrate a distinct resonance behaviour near AFMR.

A fine structure of the AFMR line near $10 \mathrm{~cm}^{-1}$ was revealed, which however dramatically disappeared while the line intensity was increased when the magnetic field was removed and the average magnetization became zero (Fig.3).

\section{THEORY AND DISCUSSION}

In order to describe and explain the observed magnetooptical effects we have calculated two dimensional transmission matrix (Jones matrix) $\hat{\mathbf{M}}$ of the $\mathrm{YFeO}_{3}$ c-cut plane-parallel plate, which determines the transmitted electric field $\mathbf{E}_{\mathrm{tr}}$ via the incident radiation $\mathbf{E}_{\text {inc }}$ :

$$
\mathbf{E}_{\text {tr }}=\hat{\mathbf{M}} \mathbf{E}_{\text {inc }}, \quad \hat{\mathbf{M}}=(1+\hat{\mathbf{R}})\left[1-(\hat{\mathbf{S}} \hat{\mathbf{R}})^{2}\right]^{-1} \hat{\mathrm{S}}(1-\hat{\mathbf{R}}),
$$

where $\hat{S}$ is a propagation matrix in the infinity $\mathrm{YFeO}_{3}$ crystal and $\hat{R}$ is a reflection matrix of the vacuum - crystal boundary. The matrix $\hat{S}$ components are determined by the sum of contributions of the two normal modes $\sim \exp \left(-\mathrm{ik}_{1,2} \mathrm{z}\right)$ of the ellipsoidal polarization having wave vectors

$$
\mathrm{k}_{1,2}=(2 \pi v / \mathrm{c})\left[\Delta_{\varepsilon \mu} \pm\left(\Delta_{\varepsilon \mu}{ }^{2}-\Delta_{\varepsilon} \Delta_{\mu}\right)^{1 / 2}\right]^{1 / 2} \text {, }
$$

where $\Delta_{\varepsilon \mu}=\left(\varepsilon_{x x} \mu_{y y}+\varepsilon_{y y} \mu_{x x}+2 \varepsilon_{x y} \mu_{x y}\right) / 2, \Delta_{\varepsilon}=\varepsilon_{x x} \varepsilon_{y y}-\varepsilon_{x y}{ }^{2}, \Delta_{\mu}=\mu_{x x} \mu_{y y}-\mu_{x y}{ }^{2} ; \varepsilon_{j k}$ and $\mu_{j k}$ are the components of the permittivity and permeability matrixes. Resonance behaviour of the $\mu_{\mathrm{jk}}(v)$ was described by $[1,2]$

$$
\mu_{x x}=1+4 \pi \chi_{\text {rot }} N(v), \quad \mu_{y y}=1+4 \pi \chi_{\perp} N(v), \quad \mu_{x y}=4 \pi\left(v / v_{0}\right)\left(\chi_{\text {rot }} \chi_{\perp}\right)^{1 / 2} \mathrm{~N}(v),
$$

where $N(v)=v_{0}^{2} /\left(v_{0}^{2}-v^{2}+i v \Delta v\right), v_{0}$ is the AFMR resonance frequency, $\Delta v$ is a linewidth, $\chi_{\text {rot }}$ and $\chi_{\perp}$ are rotation and perpendicular susceptibilities of the $\mathrm{YFeO}_{3}$ respectively. Spectra of the $T(v)$, $\rho(v)$ and $\alpha \mathrm{F}(v)$ calculated by means of the M matrix are plotted in the Fig. 1-3 and show good agreement with the experiment. The corresponding fitting parameters are: $\quad \varepsilon_{x x}{ }^{\prime}=24.0, \quad \varepsilon_{x x} \prime=0.04, \quad \varepsilon_{y y}{ }^{\prime}=21.8, \quad \varepsilon_{y y}{ }^{\prime \prime}=0.04, \quad \varepsilon_{x y} \approx 0, \quad v_{0}=9.875 \mathrm{~cm}^{-1}, \quad \Delta v=0.028 \mathrm{~cm}^{-1}$, $\chi_{\text {rot }}=10^{-4}, \chi_{\perp}=0.710^{-4}$; they were found using only $T(v)$ spectra at efla and eflb at $\alpha_{A}=0$. An unusual effect of the weak magnetic field along magnetic moment $(\mathrm{H}|| \mathrm{m}|| \mathrm{c}$-axis) on the line-shape and its intensity (Fig.3) is explained by the interference of the two normal modes of the radiation possessing strongly different propagation constants in the crystal near AFMR. In the case of $H=0$, when the sample is demagnetised and an average value of $\mu_{x y} \approx 0$, the interference disappears and the resonance line looks like a single line. The theoretical calculations and computer simulations confirmed this conclusion and allowed a good agreement between theory and experiment (Fig.3).

\section{Acknowledgements}

This work was supported in part by the Russian Foundation for Basic Researches (grant N96-02-18091) and the Ministry of Science and Technological Policy of the Russian Federation.

\section{References}

[1] Balbashov A.M., Kozlov G.V., Mukhin A.A., ProkhorovA.S., in the book "High frequency processes in magnetic materials", ed. G. Srinivasan and A. Slavin, 1995, "World Scientific", Singapore 56-98.

[2] Kocharyan K.N., Martirosyan R.M., Prpryan V.G., Sarkisyan A.L., JETP, 86 (1984), 639-643. 